\title{
Generalized Quadrangles from a Local Point of View
}

\author{
Koen Thas
}

Dedicated to the memory of professor Adriano Barlotti

\begin{abstract}
In this lecture, I will survey several recent results in the local theory of generalized quadrangles. Starting with a short introduction to the global automorphism theory, I will motivate as such the local viewpoint, and overview some of the most important local properties which are investigated nowadays. Recent results on skew translation quadrangles and forms will be described, including a solution of a question of Payne which generalizes work of Havas et al. [20, 19], and then I will mention parts of a classifcation of skew translation quadrangles which is being prepared by the author. Finally, I will consider conditions which are both global and local.
\end{abstract}

Mathematics Subject Classification (2010). 05B25, 05E18, 20B10, 20B25, 20D15, 51E12.

\section{Contents}

1. Introduction 2

2. Some combinatorics 2

3. Global viewpoint 3

4. Local viewpoint 5

\begin{tabular}{lll}
\hline 5. & Classification of STGQs & 6
\end{tabular}

\begin{tabular}{lll}
\hline 6. & STGQs and forms & 7
\end{tabular}

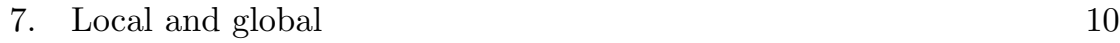

\begin{tabular}{ll}
\hline References & 14
\end{tabular}

The author is a Postdoctoral Fellow of the Fund for Scientific Research — Flanders (Belgium). 


\section{Introduction}

Generalized quadrangles were introduced by Tits in [48] as a subclass of the generalized polygons, the natural geometric modules of the groups of Lie type of (relative) rank 2. Tits showed that generalized polygons are the corner stones of buildings, and in a famous work [49, he classified all spherical buildings of rank at least 3. Classifying the rank 2 examples - which are exactly the generalized polygons - is not possible due to the existence of free constructions, so there one has to impose extra properties in order to pursue such a goal. In higher ranks, Tits showed that buildings satisfy the so-called "Moufang condition", so a natural approach would be to endow the rank 2 examples with this property. It is certainly not the goal here to survey the (global) Moufang condition (this has been done elsewhere in much detail). Let us just mention that all Moufang polygons were classified by Fong and Seitz [13, 14] in the finite case, and by Tits and Weiss [50] in general. (Some more information will also appear below.) Endowing a generalized polygon with a strong condition such as the Moufang condition does not come at little cost: the high amount of symmetry which is the result leads to the classical examples (associated to classical or algebraic groups). Even in the finite case, and especially for the gonalities three and four, the fact that many nonclassical examples exist - examples which do not admit any global group action at all — asks for the need of more general conditions or theories in which these examples can also be described, and perhaps classified. In the planar case, the introduction of translation planes was a good example of such a condition. A Moufang plane is easily shown to be a translation plane for any line, and in fact, the translation plane definition precisely expresses the fact that the plane is Moufang "at the translation line".

In this paper, we will consider properties for generalized quadrangles which express the fact that locally, the quadrangle is Moufang.

\section{Some combinatorics}

For definitions not given here, we refer to the monographs [32, 37].

A generalized quadrangle (GQ) of order $(s, t)$ is a point-line incidence structure $\mathscr{S}=(\mathscr{P}, \mathscr{B}, \mathrm{I})$ in which $\mathscr{P}$ and $\mathscr{B}$ are disjoint (nonempty) sets of objects called points and lines respectively, and for which I is a symmetric point-line incidence relation satisfying the following axioms:

- two distinct points are incident with at most one line;

- each point is incident with $t+1$ lines $(t \geq 1)$;

- each line is incident with $s+1$ points $(s \geq 1)$;

- if $p$ is a point and $L$ is a line not incident with $p$, then there is a unique point-line pair $(q, M)$ such that $p \mathrm{I} M \mathrm{I} q \mathrm{I} L$.

There is a map $D$ which sends a $\mathrm{GQ} \mathscr{S}=(\mathscr{P}, \mathscr{B}, \mathrm{I})$ of order $(s, t)$ to $\mathscr{S}^{D}=$ $(\mathscr{B}, \mathscr{P}, \mathrm{I})$, a $\mathrm{GQ}$ of order $(t, s)$ which is called the point-line dual of $\mathscr{S}$. The presence of this map is called "point-line duality"; for GQs of order $(s, t)$, in 
any definition or theorem the words "point" and "line" can be interchanged and also the parameters $s$ and $t$, to obtain the dualized version.

Let $\mathscr{S}=(\mathscr{P}, \mathscr{B}, \mathrm{I})$ be a finite generalized quadrangle of order $(s, t)$, and suppose $p \mp L,(p, L) \in \mathscr{P} \times \mathscr{B}$. Then by $\operatorname{proj}_{L} p$, we denote the unique point on $L$ collinear with $p$. Dually, $\operatorname{proj}_{p} L$ is the unique line incident with $p$ concurrent with $L$. Let $A \subseteq \mathscr{P}$; then by $A^{\perp}$ we mean $\cap_{a \in A} a^{\perp}$ (here $a^{\perp}$ is the set of points of $\mathscr{P}$ collinear with $a$, including $a$ ). We write $A^{\perp \perp}$ for $\left(A^{\perp}\right)^{\perp}$. Clearly, if $x$ and $y$ are noncollinear points, $\left|\{x, y\}^{\perp \perp}\right| \leq t+1$; if equality holds, we say that $\{x, y\}$ is regular. The point $x$ is regular provided $\{x, y\}$ is regular for every $y \in \mathscr{P} \backslash x^{\perp}$.

If the order of $\mathscr{S}$ is $\left(t^{2}, t\right)$, and $\{U, V, W\}$ is a line triad, that is, three distinct lines mutually nonintersecting, then $\left|\{U, V, W\}^{\perp}\right|=t+1$ [32, 1.2.4].

\section{Global viewpoint}

In the global viewpoint, we consider rank 2 geometries $\mathscr{M}=(\mathscr{P}, \mathscr{B}, \mathrm{I})$ that admit an automorphism group $K \leq \operatorname{Aut}(\mathscr{M})$ which acts transitively on a set $\mathscr{C}$ of substructures of $\mathscr{S}$ of the same isomorphism type. (Recall that an automorphism of $\mathscr{M}$ is a permutation of $\mathscr{P} \cup \mathscr{B}$ which preserves $\mathscr{P}, \mathscr{B}$ and incidence. The set of all automorphisms naturally forms a group by composition, and is denoted as $\operatorname{Aut}(\mathscr{M})$.) More precisely, we want $\mathscr{C}$ to consist of subgeometries of $\mathscr{M}$ such that for $\Gamma, \Gamma^{\prime} \in \mathscr{M}, \Gamma$ is isomorphic to $\Gamma^{\prime}$, and if $\Gamma^{\prime \prime}$ is a subgeometry of $\mathscr{M}$ isomorphic to any member of $\mathscr{C}$, we require it also to be a member of $\mathscr{C}$. (Note that the isomorphisms are initially only defined between the members of $\mathscr{C}-a$ priori they are not related to automorphisms of $\mathscr{M}$.) To make sense of this definition, one also wants to require that the elements of $\mathscr{C}$ are "sufficiently general"; good examples are $\mathscr{C}=\mathscr{P}, \mathscr{C}=\mathscr{B}$, $\mathscr{C}=\{(u, V) \mid(u, V) \in \mathrm{I}\}$, or $\mathscr{C}=\{$ (ordered) subquadrangles of order $(1,1)\}$, etc.

\subsection{The Moufang condition}

Already in the 1960's, Tits started a program to obtain all Moufang generalized $n$-gons, and much later, Tits and Weiss [50] eventually finished the classification of (finite and infinite) Moufang generalized $n$-gons. For the finite case, this result was already obtained by Fong and Seitz in [13, 14, the most difficult case being the case $n=4$ by far, and for this latter case, there is also a geometrical proof which is a culmination of work by Payne and Thas 32 , Chapter 9], Kantor [23] and the author [38. We refer to the author and Van Maldeghem 46] for a survey on old an new results on Moufang generalized quadrangles. We also refer to [37, Chapter 11], and especially [36] on that matter.

After the work of Fong and Seitz, the importance of local Moufang conditions became obvious - not only numerous characterizations of known classes of generalized quadrangles came out, also the theory of translation generalized quadrangles defined below essentially arose from it, and the abstraction to 
elation generalized quadrangles eventually lead to many new classes of generalized quadrangles.

\subsection{Moufang quadrangles}

A root of a $\mathrm{GQ} \mathscr{S}=(\mathscr{P}, \mathscr{B}, \mathrm{I})$ is a triple

$$
(x, L, y) \in \mathscr{P} \times \mathscr{B} \times \mathscr{P} \text { for which } x \mathrm{I} L \mathrm{I} y \text { and } x \neq y \text {. }
$$

The dual notion is a dual root. A root-elation with root $(x, L, y)=\gamma$ is an element $\alpha$ of $\operatorname{Aut}(\mathscr{S})$ which fixes $x$ and $y$ linewise, and $L$ pointwise. We also write $\alpha \in \operatorname{Aut}(\mathscr{S})_{[\gamma]}$, or $\alpha \in \operatorname{Aut}(\mathscr{S})_{[(x, L, y)]}$. So $\operatorname{Aut}(\mathscr{S})_{[\gamma]}$ is the group of all root-elations with root $\gamma$. By [32, 8.1.1], $\alpha$ cannot fix points of $\mathscr{P} \backslash L$, nor lines of $\mathscr{B} \backslash L^{\perp}$. The same can easily be proven in the infinite case. Let $U \neq L$ and $U \mathrm{I} u$, where $u$ is one of $x, y$. The root $\gamma$ is Moufang if $\operatorname{Aut}(\mathscr{S})_{[\gamma]}$ acts transitively on the points of $U \backslash\{u\}$. We define dual Moufang roots dually.

A GQ is Moufang if all roots and dual roots are Moufang. We already mentioned that all Moufang quadrangles are classified by Tits and Weiss [50].

Let $\Gamma$ be a thick, not necessarily finite, Moufang quadrangle, and let $x$ be any point of $\Gamma$. Let $z$ be a point which is not collinear with $x$, and let $U$ and $V$ be distinct lines incident with $x$. Let $u=\operatorname{proj}_{U} z$ and $v=\operatorname{proj}_{V} z$. Let $A$ be the group of root-elations with root $(x, U, u), B$ the group of root-elations with root $(x, V, v)$, and $C$ the group of dual root-elations with dual root $(U, x, V)$. Define

$$
K:=\langle A, B, C\rangle .
$$

Let $\alpha \in A$ and $\gamma \in C$; then $[\alpha, \gamma]=\alpha^{-1} \gamma^{-1} \alpha \gamma \in A \cap C=\{\mathbf{1}\}$. So $A C$ and $B C$ are (normal) subgroups of $K$, and $K=A B C$. Note that $K / A C$ acts sharply transitively on $U \backslash\{x\}$ and similarly, $K / B C$ acts sharply transitively on $V \backslash\{x\}$. So $K$ is a group of elations with center $x$; if $\ell \in K$ would fix some point $y \nsim x$, it would have to fix $\operatorname{proj}_{U} y$ and $\operatorname{proj}_{V} y$, so that $U$ and $V$ are fixed pointwise by $\ell$. As $\ell$ fixes $x$ (and $y$ ) linewise, it must be the identity. The fact that $K$ acts transitively on the points not collinear with $x$ is left to the reader as an easy exercise.

\subsection{The Knarr condition}

Although we have shown that $U$ and $V$ indeed define an elation group $K$ forcing $\Gamma^{x}$ to be a so-called "elation quadrangle" (formally defined in the next section), there is not much indication to think that the same group would be obtained for different $U$ and $V$. (Still, if $W(x)$ is the group of elements in Aut( $\Gamma)$ fixing $x$ linewise, then $K \unlhd W(x)$.) If $\Gamma$ is a Moufang quadrangle, the group $M(x)$ generated by all root-elations and dual root-elations with (dual) root containing $x$ is known to be an elation group, so in that case, we have a "canonical" way to associate an elation quadrangle to each point of the GQ. But in general, it is not clear as to whether the group $M(x)$ couldn't be larger. In the finite case, the answer was obtained by the author:

Theorem 3.1 (K. Thas [4]). Let $\Gamma$ be a finite GQ with a point $x$ such that any root and dual root containing $x$ is Moufang. Define $M(x)$ as above. Then $M(x)$ fixes all lines incident with $x$ and acts sharply transitively on the points 
not collinear with $x$; moreover, $M(x)$ is a $p$-group and so the parameters of $\Gamma$ are powers of $p$.

Norbert Knarr 25] was the first to consider the local Moufang properties considered in the previous theorem, and in fact, the theorem answered a question posed by him in the 1990's [25]. In the infinite case, the question remains unsolved, although much progress has been recently made by the author.

\section{Local viewpoint}

To incorporate as many classes of examples as possible in one unified theory, we introduce the "local point of view": impose local (group theoretical) conditions on, in this case, a generalized quadrangle, such that (hypothetical) classification would lead to both classical and nonclassical examples. In particular, we think of automorphism groups fixing a point, or a line, etc., having an interesting action on the quadrangle.

\subsection{EGQs and STGQs}

For a $\mathrm{GQ} \mathscr{S}=(\mathscr{P}, \mathscr{B}, \mathrm{I})$, we call a point $x$ an elation point, if there is an automorphism group $H \leq \operatorname{Aut}(\mathscr{S})$ that fixes $x$ linewise and acts sharply transitively on $\mathscr{P} \backslash x^{\perp}$ (the group is called "elation group"). If a GQ has an elation point, it is called an elation generalized quadrangle or, shortly, "EGQ". We will frequently use the notation $\left(\mathscr{S}^{x}, H\right)$ to indicate that $x$ is an elation point with associated elation group $H$. (Sometimes we also write $\mathscr{S}^{x}$ if we don't want to specify the elation group.)

Theorem 4.1 (D. Frohardt [15]). For a thick finite EGQ of order $(s, t)$ with $s \leq t$, we have that $s t$ is a prime power, so that the elation group is a $p$-group.

Suppose $\left(\mathscr{S}^{x}, H\right)=(\mathscr{P}, \mathscr{B}, \mathrm{I})$ is an EGQ of order $(s, t), s, t \in \mathbb{N} \backslash\{0,1\}$, and let $z$ be a point of $\mathscr{P} \backslash x^{\perp}$. Let $L_{0}, L_{1}, \ldots, L_{t}$ be the lines incident with $x$, and define $r_{i}$ and $M_{i}$ by $L_{i} \mathrm{I} r_{i} \mathrm{I} M_{i} \mathrm{I} z, 0 \leq i \leq t$. Put $H_{i}=H_{M_{i}}, H_{i}^{*}=H_{r_{i}}$ and $\mathbf{F}=\left\{H_{i} \mid 0 \leq i \leq t\right\}, \mathbf{F}^{*}=\left\{H_{i}^{*} \mid 0 \leq i \leq t\right\}$. Then $|H|=s^{2} t$ and $\mathbf{F}$ is a set of $t+1$ subgroups of $H$, each of order $s$. Also, for each $i, H_{i}^{*}$ is a subgroup of $H$ of order st containing $H_{i}$ as a subgroup. Note that if one chooses an other point for $z, \mathbf{F}$ and $\mathbf{F}^{*}$ rest unchanged up to conjugation by an element of $H$. The following two conditions are satisfied:

- $H_{i} H_{j} \cap H_{k}=\{\mathbf{1}\}$ for distinct $i, j$ and $k$;

- $H_{i}^{*} \cap H_{j}=\{\mathbf{1}\}$ for distinct $i$ and $j$.

If $H$ is a group of order $s^{2} t$ and $\mathbf{F}$ (respectively $\mathbf{F}^{*}$ ) is a set of $t+1$ subgroups $H_{i}$ (respectively $H_{i}^{*}$ ) of $H$ of order $s$ (respectively of order $s t$ ), and if the aforementioned conditions are satisfied, then the $H_{i}^{*}$ are uniquely defined by the $H_{i}$, and $\left(\mathbf{F}, \mathbf{F}^{*}\right)$, or just $\mathbf{F}$, is said to be a Kantor family or 4-gonal family of type $(s, t)$ in $H$. Using a (now) standard group coset geometry construction, one can then construct a GQ $\mathscr{S}\left(\mathbf{F}, \mathbf{F}^{*}\right)$ which is an EGQ with elation group $H$; moreover, if we start from $\mathscr{S}$ as above, then $\mathscr{S} \cong \mathscr{S}\left(\mathbf{F}, \mathbf{F}^{*}\right)$ [22, 28]. 
If $x$ is a regular point, it can be shown that $H$ contains a subgroup $\mathbb{S}$ of order $t$ consisting of automorphisms which fix each point of $x^{\perp}$ [45. Such automorphisms are called symmetries with center $x$, and $x$ is a center of symmetry since $t$ is the maximal amount of symmetries with center $x$. We then say that $\mathscr{S}^{x}$ is a skew translation quadrangle (STGQ). We call the STGQ central if $\mathbb{S}$ is contained in the center $Z(H)$ of $H$. As [45] points out, centrality is the key to classifying STGQs.

\subsection{TGQs}

If the elation group $H$ of an EGQ $\mathscr{S}^{x}$ is abelian, we call $\mathscr{S}^{x}$ a translation generalized quadrangle with translation group $H$ (and translation point $x$ ). In that case it can be shown that it is elementary abelian (cf. [32, Chapter 8], [37. §3.4]), and that $s \leq t$ [32, Chapter 8], 37, §3.3], if the order of $\mathscr{S}^{x}$ is $(s, t)$ and $\mathscr{S}^{x}$ is finite. Note that $H$ is uniquely defined - see [37, Theorem 3.3.10]. Let $\left(\mathbf{F}, \mathbf{F}^{*}\right)$ be the associated Kantor family, and suppose the TGQ is finite. By [32, §8.5], see also [37, §3.4], the ring of endomorphisms of $H$ preserving each element of $\mathbf{F} \cup \mathbf{F}^{*}$ is a field $\mathbb{F}_{q}$, over which $H$ can be seen as a vector space. Seeing the elements of $\mathbf{F}$ as subspaces (over $\mathbb{F}_{q}$ ) of the corresponding projective space $\mathbf{P}$, it appears that if $s \neq t$ or if $s=t$ is odd, one can construct another TGQ $\left(\mathscr{S}^{x}\right)^{*}$ by interpreting $\left(\mathbf{F}, \mathbf{F}^{*}\right)$ in the dual space of $\mathbf{P}$, see [32, Chapter 8], or [37, $33.9, \S 3.10]$. The TGQ $\left(\mathscr{S}^{x}\right)^{*}$ is the translation dual of $\mathscr{S}^{x}$, and has the same order.

\section{Classification of STGQs}

Up to the Hermitian quadrangles in 4 dimensions and Payne derived examples (which we will meet formally in the last section of this paper), all known finite generalized quadrangles are STGQs up to duality. Even more, except for the $\mathbf{T}_{3}(\mathscr{O})^{D}$ examples where $\mathscr{O}$ is a Suzuki-Tits ovoid of $\mathbf{P G}(3, q)$, all of them satisfy the Knarr condition at the elation point (see [43, 45]).

Dirk Hachenberger [18, and independently X. Chen, see [43, obtained a first step in classifying STGQs:

Theorem 5.1 (D. Hachenberger [18, X. Chen — see [4]). The parameters of a thick STGQ are powers of the same prime.

The author is preparing a classification of STGQs [45] (which will hopefully be finished soon), in which centrality is a basic feature; once one knows that the symmetries about the elation point are in the center of the elation group, an STGQ already satisfies some fairly strong Moufang conditions which permit us to develop a strong structural theory.

In odd characteristic, for instance, we already have the following result when the number of points equals the number of lines:

Theorem 5.2 (K. Thas [45]). An STGQ of odd order $s$ is isomorphic to $\mathbf{W}(s)$. 
The latter theorem has strong consequences for groups as well (characterizing semifield groups, that is, Sylow $p$-subgroups of $\mathbf{P S L}_{3}(q)$ with $q=p^{h}$ and $p$ odd). Many more results can be found in [45, but here, we restrict ourself only to the following section, which reflects the flavor of parts of [45.

\section{STGQs and forms}

In the last few decades, the most fruitful way to construct finite generalized quadrangles was through the detection of certain Kantor families (arising from a so-called " $q$-clan") in the 2-dimensional general Heisenberg group $\mathbf{H}_{2}(q)$ over some finite field $\mathbb{F}_{q}$. All these examples are so-called "flock quadrangles". In [30], Payne constructed from the Ganley flock quadrangles new quadrangles ("Roman quadrangles") which appeared not to arise from flocks, but still arise via a Kantor family construction (in some group $\mathscr{G}$ of the same order as $\mathbf{H}_{2}(q)$ ). The fundamental question (first asked by Payne in op. cit., see his excellent account [31]) then arose as to whether $\mathbf{H}_{2}(q) \cong \mathscr{G}$. In [19, Havas et al. showed that for the Roman quadrangles with parameters $(729,27)$, and using a computer program, the corresponding groups are not isomorphic. In [20, they obtained the result for all Roman quadrangles. The proof consists of showing that noncentral elements in $\mathbf{H}_{2}(q)$ and $\mathscr{G}$ have nonisomorphic centralizers.

The construction of Payne appears to be a special case of a more general one.

Theorem 6.1 (K. Thas [40]). Each flock quadrangle $\mathscr{S}=\mathscr{S}(\mathscr{F})$ for which the dual $\mathscr{S}^{D}$ is a translation generalized quadrangle gives rise to another generalized quadrangle (which is the dual of the translation dual $\left(\mathscr{S}^{D}\right)^{*}$ ) which is in general not isomorphic to $\mathscr{S}^{D}$, and which also arises from a Kantor family.

Denote the class of such flock quadrangles by $\mathscr{C}$. (By reasons to explain later, we only consider odd characteristic.)



In 42 , we resolved the question of Payne for the complete class $\mathscr{C}$, by showing that flock quadrangles are characterized by their groups (in any characteristic), a question which was open for quite some time. As an application of the main result, the special case of prime $q$ yields an alternative proof of the main result of [2].

Theorem 6.2 (K. Thas 42]). Let $\mathscr{S}$ be an EGQ of order $\left(q^{2}, q\right), q$ any prime power, with elation group $\mathbf{H}_{2}(q)$. Then $\mathscr{S}$ is a flock quadrangle.

In terms of Kantor families, this result reads as follows: 
Theorem 6.3 (K. Thas [42]). A Kantor family of type $\left(q^{2}, q\right)$ in $\mathbf{H}_{2}(q)$ arises from a $q$-clan.

Passing from the latter theorem to the solution of Payne's question goes as follows.

Theorem 6.4 (K. Thas [4]). Let $\mathscr{S}=\mathscr{S}(\mathscr{F})$ be a flock GQ of order $\left(q^{2}, q\right)$ for which the dual $\mathscr{S}^{D}$ is a TGQ, $q$ odd. Let $\mathscr{G}$ be defined as in the diagram above. Then $\mathscr{G} \cong \mathbf{H}_{2}(q)$ if and only if $\mathscr{F}$ is a Kantor-Knuth flock if and only if $\mathscr{S} \cong\left(\left(\mathscr{S}^{D}\right)^{*}\right)^{D}$.

If $\mathscr{S}(\mathscr{F})$ is the Ganley flock quadrangle, the dual of $\left(\mathscr{S}^{D}\right)^{*}$ is the Roman quadrangle (which is not isomorphic to a Kantor-Knuth quadrangle), yielding thus the result of Havas et al. [19, 20]. If $\mathscr{S}=\mathscr{S}(\mathscr{F})$ is a flock GQ of order $\left(q^{2}, q\right)$ for which the dual $\mathscr{S}^{D}$ is a TGQ, and $q$ is even, $\mathscr{S} \cong \mathbf{H}\left(3, q^{2}\right)$ (cf. N. L. Johnson [21, 6] or [37, Theorem 5.1.11]). In that case, the analogous question is reduced to the main results of [33, 39. Other implications can be found in 42 .

In the rest of this section, we provide some more details.

\subsection{The general Heisenberg group}

The general Heisenberg group $\mathbf{H}_{n}(q)$ (sometimes also written as $\mathbf{H}_{n}$ if we don't want to specify $q$ ) of dimension $2 n+1$ over $\mathbb{F}_{q}$, with $n \neq 0$ a natural number, is the group of square $(n+2) \times(n+2)$-matrices with entries in $\mathbb{F}_{q}$, of the following form (and with the usual matrix multiplication):

$$
\left(\begin{array}{ccc}
1 & \alpha & c \\
0 & \mathbf{I}_{n} & \beta^{T} \\
0 & 0 & 1
\end{array}\right),
$$

where $\alpha, \beta \in \mathbb{F}_{q}^{n}, c \in \mathbb{F}_{q}$ and with $\mathbf{I}_{n}$ being the $n \times n$-identity matrix. The group $\mathbf{H}_{n}$ is isomorphic to the group $\left\{(\alpha, c, \beta) \mid \alpha, \beta \in \mathbb{F}_{q}^{n}, c \in \mathbb{F}_{q}\right\}$, where the group operation $\circ$ is given by

$$
(\alpha, c, \beta) \circ\left(\alpha^{\prime}, c^{\prime}, \beta^{\prime}\right)=\left(\alpha+\alpha^{\prime}, c+c^{\prime}+\alpha \beta^{\prime T}, \beta+\beta^{\prime}\right) .
$$

The following properties hold for $\mathbf{H}_{n}$ (defined over $\mathbb{F}_{q}$ ).

- $\mathbf{H}_{n}$ has exponent $p$ if $q=p^{h}$ with $p$ an odd prime; it has exponent 4 if $q$ is even.

- The center of $\mathbf{H}_{n}$ is given by $Z=Z\left(\mathbf{H}_{n}\right)=\left\{(0, c, 0) \mid c \in \mathbb{F}_{q}\right\}$.

- $\left[\mathbf{H}_{n}, \mathbf{H}_{n}\right]=Z=\Phi\left(\mathbf{H}_{n}\right)$ and $\mathbf{H}_{n}$ is nilpotent of class $2\left(\Phi\left(\mathbf{H}_{n}\right)\right.$ is the Frattini subgroup of $H$, that is, the intersection of all its maximal subgroups).

The following (very important) property is less well-known [22, 24].

- Let $V$ be the elementary abelian $p$-group $\mathbf{H}_{2}(q) / Z$. The map $\chi$

$$
\chi: V \times V \mapsto \mathbb{F}_{q}:(a Z, b Z) \mapsto[a, b]
$$

defines a bilinear alternating nonsingular (BAN-) form over $\mathbb{F}_{q} \equiv Z$. So $V$ can be seen as a 4 -dimensional space over $\mathbb{F}_{q}$, and in the corresponding 
projective 3 -space over $\mathbb{F}_{q}, \chi$ defines a symplectic polar space $\mathbf{W}(q)$ of rank 2 (projective index 1 ).

Note that we already met $\mathbf{H}_{1}(q)$ in the previous section.

\subsection{Flock quadrangles and $q$-clans}

Let $\mathscr{F}$ be a flock of the quadratic cone $\mathscr{K}$ in $\mathbf{P G}(3, q)$ with equation $X_{0} X_{1}=$ $X_{2}^{2}$; so $\mathscr{F}$ is a partition of $\mathscr{K}$ without the vertex consisting of irreducible conics. Then it was noticed in [34] that the equations of the planes (with respect to a suitable reference system) generated by the conics define a Kantor family of type $\left(q^{2}, q\right)$ in $\mathbf{H}_{2}(q)$; that is, to $\mathscr{F}$ corresponds an EGQ $\mathscr{S}^{x}=$ $\mathscr{S}(\mathscr{F})$, called flock quadrangle, of order $\left(q^{2}, q\right)$ with elation group $\mathbf{H}_{2}(q)$. Also, it happens to be an STGQ with respect to $x$, and Kantor families in $\mathbf{H}_{2}(q)$ that give rise to flock quadrangles are precisely those related to "q-clans", see [6, 37].

One of the corollaries of the main result of 42 is that Kantor families in $\mathbf{H}_{2}(q)$ always are of this type.

We finally mention that if $\mathscr{S}(\mathscr{F})$ is a flock quadrangle of order $\left(q^{2}, q\right)$, and its dual is a TGQ, then $\mathscr{S}(\mathscr{F}) \cong \mathbf{H}\left(3, q^{2}\right)$ if $q$ is even. If $q$ is odd, the TGQ $\mathscr{S}(\mathscr{F})^{D}$ is isomorphic to its translation dual if and only if $\mathscr{F}$ is a KantorKnuth semifield flock. We refer to [21, 6, 37] for further details.

\subsection{Property $(G)$ and flock GQs}

Let $x$ be a point of a GQ $\mathscr{S}$ of order $\left(t^{2}, t\right)$, and let $U, V$ be distinct lines incident with $x$. Then $\mathscr{S}$ satisfies Property $(G)$ at the pair $\{U, V\}$ if any triad of lines $\{V, W, Z\}$ in $U^{\perp}$ is 3-regular, which means that

$$
\left|\{V, W, Z\}^{\perp}\right|=\left|\{V, W, Z\}^{\perp \perp}\right|=t+1 .
$$

Note that the definition is symmetric in $U$ and $V$. The flag $(x, L)$ has Property $(G)$ if all pairs $\{L, M\}$ of distinct lines incident with $x$ have Property $(\mathrm{G})$. One says that $x$ has Property $(G)$ if all pairs $\{U, V\}$ incident with $x$ have Property (G).

It can be shown that if $\mathscr{S}^{x}$ is a flock quadrangle, the point $x$ satisfies Property (G) 30 .

The following theorem was first obtained in odd characteristic in [35, answering a fundamental conjecture of Payne's essay [30. In the case of even characteristic, relying on [35, it was obtained only much later by M. R. Brown 5]. (In odd characteristic, only one flag was required in 35; later it was shown that one pair of intersecting lines was sufficient [3].)

Theorem 6.5 ([35, 5]). A GQ of order $\left(t^{2}, t\right)$ satisfying Property (G) at two distinct flags $(u, L)$ and $(u, M)$ for some point $u$ is isomorphic to a flock GQ.

\subsection{Special groups and alternating forms}

Let $H$ be a special group [16, p. 183] of order $q^{m}, m \in \mathbb{N}_{0,1}=\mathbb{N} \backslash\{0,1\}$, with $q=p^{h}$ a power of the prime $p$, for which

$$
Z(H)=\Phi(H)=[H, H]
$$


is (elementary abelian) of order $q$.

The exponent of $H$ is easily seen to be $p$ or $p^{2}$ if $p$ is odd, and 4 if $p=2$.

Define a bi-additive map $\chi$ as follows:

$$
\chi: V \times V \mapsto \mathbb{F}_{q}:(a Z(H), b Z(H)) \mapsto[a, b],
$$

where we see $H / Z(H)$ as a vector space $V$ over $\mathbb{F}_{p}$, and identify $Z(H)$ with $\mathbb{F}_{q}$. We assume that $\chi$ defines a BAN-form over $\mathbb{F}_{q} \equiv Z(H)$, that is, the commuting structure of $\chi$ is a symplectic polar space $\mathbf{W}(2 n-1, q)$ of rank $n-1$ over $\mathbb{F}_{q}$. The polar space then occurs in the projective space $\mathbf{P G}(2 n-1, q)$ associated to $V$, with $m=2 n+1$.

Observation 6.6 (K. Thas [42]). If $H$ admits a Kantor family of type $(s, q)$, then $s \in\left\{q, q^{2}\right\}$, and so $|H| \in\left\{q^{3}, q^{5}\right\}$.

The main theorem of 42 is the following.

Theorem 6.7 (K. Thas [42]). Suppose $H$ is a special $p$-group of order $q^{5}$ for which $Z(H)=\Phi(H)=[H, H]$ is elementary abelian of order $q$. Suppose $H$ admits a Kantor family of type $\left(q^{2}, q\right)$, and suppose $\chi$ defines a BAN-form over $\mathbb{F}_{q}$. Then $H \cong \mathbf{H}_{2}(q)$, and the corresponding generalized quadrangle $\mathscr{S}$ of order $\left(q^{2}, q\right)$ is a flock quadrangle.

The generalization of the theorem of Havas et al. [19, 20] was already treated in the introduction of this section.

\subsection{Elation quadrangles of order $(s, p), p$ a prime}

In the aforementioned paper [2], the following theorem, which complements the result of Bloemen et al. 4 classifying EGQs of order $(p, t), p$ a prime, was obtained.

Theorem 6.8 (Bamberg et al. 2 $)$. An EGQ $\left(\mathscr{S}^{x}, H\right)$ of order $(s, p)$ with $p$ a prime, is either isomorphic to $\mathbf{W}(p)$, or to a flock quadrangle, in which case $s=p^{2}$.

In 42, the main result is applied to obtain an alternative and very short proof of Theorem 6.8. It also corrects a mistake which is contained in a lemma of [2] (cf. 42 for details).

\section{Local and global}

In this final section, we want to consider a specific situation in which a local group action on some GQ $\Gamma$, yields a global group action on another GQ which is "derived" from $\Gamma$. By slight abuse of language it is very much alike the situation in which the local group action of the translation group of a translation plane induces a transitive action on the points of the corresponding affine translation plane. Strangely enough, the obtained global action on the derived quadrangle is by no means classical anymore (in the sense that the derived quadrangle itself is not classical). 


\subsection{Payne derived GQs}

We recall Payne's construction of GQs from [27]. Let $\mathscr{Q}$ be a finite GQ of order $s$ with a regular point $x$. Define the point-line incidence geometry $\mathscr{P}=$ $\mathscr{P}(\mathscr{Q}, x)$ as follows.

- The points of $\mathscr{P}$ are the points of $\mathscr{Q}$ not collinear with $x$.

- The lines of $\mathscr{P}$ are the lines of $\mathscr{Q}$ not through $x$ together with all sets of the form $\{x, r\}^{\perp \perp} \backslash\{x\}$, where $x \not r$.

- Incidence is the natural one.

We have the following theorem.

Theorem 7.1 (S. E. Payne [27]). The point-line geometry $\mathscr{P}$ is a GQ of order $(s-1, s+1)$.

The GQ $\mathscr{P}$ is the so-called Payne-derived GQ or Payne derivative of $\mathscr{Q}$ (with respect to $x)$. In the case where $\mathscr{Q} \cong \mathbf{W}(q)$, every point of $\mathscr{Q}$ is regular 32 , Chapter 3], and all Payne derivatives are isomorphic, independent of the regular point chosen. We will denote the Payne-derivative of $\mathbf{W}(q)$ therefore simply by $\mathscr{P}(q)$.

For a point-line geometry $\mathscr{M}=(\mathscr{P}, \mathscr{B}, \mathrm{I})$, we call a Singer group (with respect to points) a subgroup of $\operatorname{Aut}(\mathscr{M})$ which acts sharply transitively on $\mathscr{P}$. The set of all Singer groups of $\mathscr{M}$ is denoted $\mathbf{S}(\mathscr{M})$.

In 12 the known GQs admitting a Singer group were classified in a combinatorial fashion. Through a different approach, using the recent classification of regular subgroups of almost simple primitive groups [26], Bamberg and Giudici 1] also obtained the classification of classical GQs admitting a Singer group (having pointed out a small error in [12] which led to one extra example being overlooked in [12). By using [26] they independently obtained the possible Singer groups that can act on a classical GQ. In their paper they also provide some new examples of groups that act as a Singer group on $\mathscr{P}(q)$. In this final section, we mention very recent work of De Winter and the author [10], in which the authors obtain a classification of Singer groups of $\mathscr{P}(q)$, starting from a handy criterion, and showing that contrary to earlier beliefs, there are many such groups.

\subsection{Criterion}

Consider the following general situation. Suppose $\mathscr{S}$ is a thick finite GQ of order $s$, and let $x$ be a regular point of $\mathscr{S}$. Suppose $G$ is a Singer group of $\mathscr{P}(\mathscr{S}, x)$ with the following properties:

- it is induced by some automorphism group of $\mathscr{S}$, that is, by some subgroup $\bar{G}$ of $\operatorname{Aut}(\mathscr{S})_{x}$;

- $\bar{G}$ contains a group $\mathbb{S}$ of order $s$ consisting of symmetries with center $x$.

Construct the standard affine plane $\Pi(x)$ of order $s$ from the regular point $x$ [32, 1.3.1]:

- its POINTS are the sets $\{y, z\}^{\perp \perp}$ with $z \not y, z, y \in x^{\perp}$,

- its LINES are the elements of $x^{\perp} \backslash\{x\}$. 
Note that elements of $\operatorname{Aut}(\mathscr{S})_{x}$ induce elements of $\operatorname{Aut}(\Pi(x))$ via the following map:

$$
\xi: \operatorname{Aut}(\mathscr{S})_{x} \mapsto \operatorname{Aut}(\Pi(x)): g \mapsto g \mathbb{S} .
$$

Note also that $\operatorname{Aut}(\mathscr{S})_{x}$ faithfully induces a subgroup of $\operatorname{Aut}(\mathscr{P}(\mathscr{S}, x))$.

Theorem 7.2 (S. De Winter and K. Thas [10]). We have that $\bar{G} / \mathbb{S}$ is an automorphism group of $\Pi(x)$ which acts sharply transitively on its points. Vice versa, let $K$ be a subgroup of $\operatorname{Aut}(\Pi(x))$ which acts sharply transitively on its points, and suppose it is induced by some automorphism group $K^{\#}$ of $\mathscr{S}$ (which then has to fix $x$ ). Then $\bar{K}=\left\langle\mathbb{S}, K^{\#}\right\rangle$, the group obtained by adjoining $\mathbb{S}$ to $K^{\#}$, induces a Singer group of $\mathscr{P}(\mathscr{S}, x)$.

In particular, if $K$ is a translation group of $\Pi(x)$, then $\bar{K}$ is a (complete) elation group for $\mathscr{S}^{x}$. (Vice versa, an elation group of $\mathscr{S}^{x}$ containing $\mathbb{S}$ induces a translation group of $\Pi(x)$.) If $K$ is as such, $s$ is of course a prime power. In general, automorphisms of Payne-derived GQs are not induced by automorphisms of the ambient GQ - cf. the discussion in [8]. For the classical case, we have the following satisfying situation.

Theorem 7.3 (T. Grundhöfer, M. Joswig and M. Stroppel [17]). If $q \geq 5$, any automorphism of $\mathscr{P}(q)$ is induced by an automorphism of $\mathbf{W}(q)$ fixing $x$.

All known (counter) examples of automorphisms not coming from the ambient GQ exist in even characteristic (besides some small sporadic examples in odd characteristic), see [8]. The most general result available is the following:

Theorem 7.4 (S. De Winter and K. Thas [8]). Let $\mathscr{P}(\mathscr{S}, x)$ be Payne derived from the thick finite GQ $\mathscr{S}$ of order $s$, with $s$ odd and $s \geq 5$. If $x$ is a center of symmetry, then any automorphism of $\mathscr{P}(\mathscr{S}, x)$ is induced by an element of $\operatorname{Aut}(\mathscr{S})_{x}$.

Let $\mathscr{S}$ be a GQ satisfying the conditions of the previous theorem, and suppose $K$ is a Singer group of $\mathscr{P}(\mathscr{S}, x)$. Then by Theorem 7.4 , and the criterion of the previous paragraph, $K$ is induced by an automorphism group $\bar{K}$ of $\mathscr{S}$ of order $s^{3}$ which induces a point-regular automorphism group of $\Pi(x)$.

\subsection{Classical case}

Let $K$ be a Singer group of $\mathscr{P}(q)$ inducing a sharply transitively group on the points of $\Pi(x)$, that is, let $K \in \mathbf{S}^{\mathbb{S}}(\mathscr{P}(q))$ (in the notation of [10]). In [10] the authors classified those $K$ (or $\bar{K}$ ) for which the quotient $\bar{K} / \mathbb{S}$ is abelian. We provide some more details for the general case. First consider $\bar{K}$; since it is a $p$-group, it fixes some line $L I x$, whence $\bar{K} / \mathbb{S}=T$ fixes a flag at infinity of $\Pi(x)$, say $(\ell,[\infty])$, where $[\infty]$ is the line at infinity. We want to consider those $T$ which are contained in the group $H(\ell) \cong \mathbf{H}_{1}(q)$ which is generated by the translation group $A$ "of" $[\infty]$ and the translation group $B$ "of" $\ell$. (If $T$ is abelian, this is always the case.) We will see in the next paragraph that this assumption is natural, and that in many cases it is not even an extra assumption. 
Theorem 7.5 (S. De Winter and K. Thas [10). There is a natural injection $\eta$ from the set $\mathbf{B}(\ell)$ of subgroups of $H(\ell) / Z(H(\ell))$ of order $q$ which intersect trivially with $B / Z(H(\ell))$ to $\mathbf{S}^{\mathbb{S}}(\mathscr{P}(q))$.

Note that when $q$ is odd, precisely $q$ elements of $\mathbf{B}(\ell)$ give rise to elements of $\mathbf{S}^{\mathbb{S}}(\mathscr{P}(q))$ with abelian quotients in $\mathbf{S}(\Pi(x)$ ) (one of these elements yielding the translation group of $\Pi(x)$ and so the elation group of $\left.\mathbf{W}(q)^{x}\right)$; when $q$ is even, precisely one element does.

Corollary 7.6 (S. De Winter and K. Thas [10]). We have that

$$
|\mathbf{B}(\ell)|=\frac{p^{h^{2}} \prod_{i=h+1}^{2 h-1}\left(p^{i}-1\right)}{p-1} \leq|\mathbf{S}(\mathscr{P}(q))| .
$$

The number of Singer groups with nonabelian quotients of $\mathscr{P}(q)$ coming from $\eta(\mathbf{B}(\ell))$ is

$$
\frac{p^{h^{2}} \prod_{i=h+1}^{2 h-1}\left(p^{i}-1\right)}{p-1}-q^{p} \quad \bmod 2
$$

Here $\frac{p^{h^{2}} \prod_{i=h+1}^{2 h-1}\left(p^{i}-1\right)}{p-1}$ evaluated at $h=1$ is set to be $p$.

Letting $\ell$ vary on $[\infty]$, and noting that $\left|\mathbf{B}(\ell) \cap \mathbf{B}\left(\ell^{\prime}\right)\right|=1$ for $\ell \neq \ell^{\prime}$, we have that the total number of Singer groups with nonabelian quotients of $\mathscr{P}(q)$ coming from $\eta\left(\bigcup_{\ell} \mathbf{B}(\ell)\right)$ is

$$
1+(q+1)\left(\frac{p^{h^{2}} \prod_{i=h+1}^{2 h-1}\left(p^{i}-1\right)}{p-1}-1\right)-((q+1) q)^{p} \bmod 2 .
$$

\subsection{Linear Singer groups, and the case $(p, h)=1$}

Suppose that $q \geq 5$; then each Singer group of $\mathscr{P}(q)$ is induced by a subgroup of $\operatorname{Aut}(\mathbf{W}(q))_{x}$. Let $K$ be a linear Singer group of $\mathscr{P}(q)$ (that is, induced by some subgroup of $\left.\mathbf{P G S p}{ }_{4}(q)_{x}\right)$. Then one observes that $\bar{K} /(\bar{K} \cap \mathbb{S})$ is a subgroup of $H(\ell)$ for some $\ell \mathrm{I}[\infty]$, and

$$
(\bar{K} /(\bar{K} \cap \mathbb{S})) \cap B \leq Z(H(\ell)) .
$$

So $\mathbb{S} \leq \bar{K}$, and whence the map $\eta$ defines a bijection from $\cup_{\ell} \mathbf{B}(\ell)$ to the linear Singer groups of $\mathscr{P}(q)$. Corollary 7.6 gives the precise numerical information. If $(p, h)=1$, any Singer group of $\mathscr{P}(q)$ is linear, so that we have a complete classification.

Theorem 7.7 (S. De Winter and K. Thas 10]). When $q \geq 5$, all linear Singer groups are essentially known; in particular, if $(p, h)=1$, we have a complete classification of Singer groups of $\mathscr{P}\left(p^{h}\right)$. 


\section{References}

[1] Bamberg, J., Giudici, M.: Point regular automorphism groups of generalized quadrangles. Preprint.

[2] Bamberg, J., Penttila, T., Schneider, C.: Elation generalized quadrangles for which the number of lines on a point is the successor of a prime. J. Aust. Math. Soc. 85, 289-303 (2008).

[3] Barwick, S.G., Brown, M.R., Penttila, T.: Flock generalized quadrangles and tetradic sets of elliptic quadrics of $\mathrm{PG}(3, q)$. J. Combin. Theory Ser. A 113, 273-290 (2006).

[4] Bloemen, I., Thas, J.A., Van Maldeghem, H.: Elation generalized quadrangles of order $(p, t), p$ prime, are classical. Special issue on orthogonal arrays and affine designs, Part I, J. Statist. Plann. Inference 56, 49-55 (1996).

[5] Brown, M.R.: Projective ovoids and generalized quadrangles. Adv. Geom. 7, 65-81 (2007).

[6] Cardinali, I., Payne, S.E.: q-Clan Geometries in Characteristic 2. Frontiers in Mathematics, Birkhäuser Verlag, Basel, 2007.

[7] De Winter, S., Thas, K.: Generalized quadrangles with an abelian Singer group. Des. Codes Cryptogr. 39, 81-87 (2006).

[8] _ : The automorphism group of Payne derived generalized quadrangles. Adv. Math. 214, 146-156 (2007).

[9] __ Generalized quadrangles admitting a sharply transitive Heisenberg group. Des. Codes Cryptogr. 47, 237-242 (2008).

[10] _ : A criterion concerning Singer groups of generalized quadrangles. 15pp., Submitted.

[11] De Winter, S., Shult, E.E., Thas, K.: Singer Quadrangles, Oberwolfach Preprint OWP 2009-07, 2009.

[12] _ : The Singer quadrangles amongst the known generalized quadrangles. 40pp., Submitted.

[13] Fong, P., Seitz, G.M.: Groups with a $(B, N)$-pair of rank 2, I. Invent. Math. 21, 1-57 (1973).

[14] _ : Groups with a (B,N)-pair of rank 2, II. Invent. Math. 24, 191-239 (1974).

[15] Frohardt, D.: Groups which produce generalized quadrangles. J. Combin. Theory Ser. A 48, 139-145 (1988).

[16] Gorenstein, D.: Finite Groups. Second edition. Chelsea Publishing Co., New York, 1980.

[17] Grundhöfer, T., Joswig, M., Stroppel, M.: Slanted symplectic quadrangles. Geom. Dedicata 49, 143-154 (1994).

[18] Hachenberger, D.: Groups admitting a Kantor family and a factorized normal subgroup. Special issue dedicated to Hanfried Lenz, Des. Codes Cryptogr. 8, 135-143 (1996).

[19] Havas, G., Leedham-Green, C.R., O'Brien, E.A., Slattery, M.C.: Computing with elation groups. Finite Geometries, Groups, and Computation, 95-102, Walter de Gruyter GmbH \& Co. KG, Berlin, 2006.

[20] __ Certain Roman and flock generalized quadrangles have nonisomorphic elation groups. Adv. Geom. 26, 389-396 (2006). 
[21] Johnson, N.L.: Semifield flocks of quadratic cones. Simon Stevin 61, 313-326 (1987).

[22] Kantor, W. M.: Generalized quadrangles associated with $G_{2}(q)$. J. Combin. Theory Ser. A 29, 212-219 (1980).

[23] _ _ Automorphism groups of some generalized quadrangles. Advances in Finite Geometries and Designs, Edited by J. W. P. Hirschfeld et al., Proceedings of the Third Isle of Thorns Conference 1990, Oxford University Press, New York, 251-256 (1991).

[24] _ Generalized quadrangles, flocks and BLT sets. J. Combin. Theory Ser. A 58, 153-157 (1991).

[25] Knarr, N.: Private Communication, October 2004 and June 2005.

[26] Liebeck, M.W., Praeger, C.E., Saxl, J.: Regular subgroups of primitive permutation groups. Mém. Amer. Math. Soc. 203 (2010).

[27] Payne, S.E.: Non-isomorphic generalized quadrangles. J. Algebra 18, 201-212 (1971).

[28] _ _ Generalized quadrangles as group coset geometries. Proceedings of the Eleventh Southeastern Conference on Combinatorics, Graph Theory and Computing (Florida Atlantic Univ., Boca Raton, Fla., 1980), Vol II, Congr. Numer. 29, 717-734 (1980).

[29] _ _ A garden of generalized quadrangles. Proceedings of the conference on groups and geometry, Part A (Madison, Wis., 1985), Algebras Groups Geom. 2, 323-354 (1985).

[30] _ _ An essay on skew translation generalized quadrangles. Geom. Dedicata 32, 93-118 (1989).

[31] _ Finite groups that admit Kantor families. Finite Geometries, Groups, and Computation, 191-202, Walter de Gruyter GmbH \& Co. KG, Berlin, 2006.

[32] Payne, S.E., Thas, J.A.: Finite Generalized Quadrangles. Second edition, EMS Series of Lectures in Mathematics, European Mathematical Society, 2009.

[33] Rostermundt, R.: Elation groups of the Hermitian surface $H\left(3, q^{2}\right)$ over a finite field of characteristic 2. Innov. Incidence Geom. 5, 117-128 (2007).

[34] Thas, J.A.: Generalized quadrangles and flocks of cones. European J. Combin. 8, 441-452 (1987).

[35] _ Generalized quadrangles of order $\left(s, s^{2}\right)$, III. J. Combin. Theory Ser. A 87, 247-272 (1999).

[36] Thas, J.A., Thas, K., Van Maldeghem, H.: Moufang Quadrangles: Characterizations, Classification, Generalizations, Capita Selecta in Geometry - A series of 27 lectures, i+87pp., Ghent University, Ghent, Spring 2003.

[37] _ : Translation Generalized Quadrangles, Series in Pure Mathematics 26, World Scientific Publishing Co. Pte. Ltd., Hackensack, NJ, 2006.

[38] Thas, K.: Automorphisms and characterizations of finite generalized quadrangles. Generalized Polygons, Proceedings of the Academy Contact Forum "Generalized Polygons" 20 October, Palace of the Academies, Brussels, Belgium, Edited by F. De Clerck et al., Universa Press, 111-172 (2001).

[39] _ Some basic questions and conjectures on elation generalized quadrangles, and their solutions. Bull. Belg. Math. Soc. — Simon Stevin 12, 909-918 (2005). 
[40] _ : A question of Kantor on elations of dual translation generalized quadrangles. Adv. Geom. 7, 375-378 (2007).

[41] _ Solution of a question of Knarr. Proc. Amer. Math. Soc. 136, 14091418 (2008).

[42] _ _ Isomorphisms of groups related to flocks. 12pp., Submitted.

[43] _ _ Lectures on Elation Quadrangles. monograph, 135 pp., Submitted.

[44] _ : Order in building theory. 110 pp., Submitted.

[45] __ Central aspects of skew translation generalized quadrangles. 140 pp., Preprint.

[46] Thas, K., Van Maldeghem, H.: Moufang-like conditions for generalized quadrangles and classification of all quasi-transitive generalized quadrangles. Discrete Math. 294, 203-217 (2005).

[47] _ : Geometric characterizations of Chevalley groups of type $\mathbf{B}_{2}$. Trans. Amer. Math. Soc. 360, 2327-2357 (2008).

[48] Tits, J.: Sur la trialité et certains groupes qui s'en déduisent. Inst. Hautes Etudes Sci. Publ. Math. 2, 13-60 (1959).

[49] _ B Buildings of Spherical Type and Finite BN-Pairs. Lecture Notes in Mathematics 386, Springer-Verlag, Berlin-New York, 1974.

[50] Tits, J., Weiss, R.M.: Moufang Polygons. Springer Monographs in Mathematics, Springer-Verlag, Berlin, 2002.

[51] Van Maldeghem, H.: Generalized Polygons. Monographs in Mathematics 93, Birkhäuser-Verlag, Basel, 1998.

Koen Thas

Ghent University, Department of Mathematics, Krijgslaan 281, S25, B-9000 Ghent, Belgium

e-mail: kthas@cage.UGent.be 